\title{
A FOURTH ORDER ELLIPTIC EQUATION WITH NONLINEAR BOUNDARY CONDITIONS
}

\author{
JULIÁN FERNÁNDEZ BONDER AND JULIO D. ROSSI
}

\begin{abstract}
In this paper we study the existence of infinitely many nontrivial solutions of the following problem, $-\Delta^{2} u=u$ in $\Omega,-\frac{\partial \Delta u}{\partial \nu}=f(x, u)$ on $\partial \Omega$, and either $\frac{\partial u}{\partial \nu}=0$ or $\Delta u=0$ on $\partial \Omega$. We assume that $f(x, u)$ is superlinear and either subcritical or a sublinear perturbation of the critical case. For the proof in the critical case we apply the concentration compactness method.
\end{abstract}

\section{INTRODUCTION.}

In this paper we study the existence of nontrivial solutions of the following fourth order problem,

$$
\left\{\begin{aligned}
-\Delta^{2} u & =u & & \text { in } \Omega, \\
-\frac{\partial \Delta u}{\partial \nu} & =f(x, u) & & \text { on } \partial \Omega .
\end{aligned}\right.
$$

Here $\Omega$ is a bounded smooth domain in $\mathbb{R}^{N}, \frac{\partial}{\partial \nu}$ is the outer normal derivative and $f: \partial \Omega \times \mathbb{R} \times \mathbb{R} \rightarrow \mathbb{R}$ is a smooth function with some precise assumptions that we will state below. Also we impose one of the following boundary conditions

$$
\Delta u=0 \quad \text { on } \partial \Omega,
$$

or

$$
\frac{\partial u}{\partial \nu}=0 \quad \text { on } \partial \Omega .
$$

Existence results for nonlinear elliptic problems of fourth order have deserved a great deal of interest (see for example [6]). In the semilinear case see the references $[3],[7]$ and [14] for some existence results. See also [15] for some nonexistence results also in the semilinear case. However the nonlinear boundary conditions are less covered in the literature (see for example [4], [10] for the Laplace equation in a half space). The aim of this work is to show how the usual variational techniques can be extended to deal with fourth order problems with nonlinear boundary conditions. So, we address the existence problem with a nonlinear boundary condition for the bilaplacian operator.

In this work we find infinitely many weak solutions for problems (1.1)-(1.2) and (1.1)-(1.3) under suitable assumptions on the nonlinearity $f$. First, we consider a subcritical superlinear nonlinearity $f$. To handle this case, we apply abstract results from critical point theory, some topological tools and Sobolev trace inequalities to deal with the boundary terms. The proofs are rather elementary, but we include

Key words and phrases. bilaplacian, nonlinear boundary conditions, variational problems 2000 Mathematics Subject Classification. 35J35, 35J65, 35J40.

Partially supported by Universidad de Buenos Aires under grant TX047, by ANPCyT PICT No. 03-00000-00137 and by CONICET, PIP0660/98. J.D. Rossi is also partially supported by Fundación Antorchas. 
a sketch for the sake of completeness. Next, we study a critical nonlinearity with a sublinear perturbation. This is the main part of the paper. For this case, our results are inspired by [3] where the authors study a similar nonlinearity in the semilinear case. The method that we apply here is the concentration compactness method combined with topological arguments. We remark that the results proved here can be extended to handle equations like $-\Delta^{2} u=u+g(x, u)$ with the same boundary conditions. To clarify the exposition we will state and prove our results for (1.1)-(1.3) and (1.1)-(1.2), leaving the details to the reader for the general case using similar hypothesis on $g$ that the ones used here on $f$.

For weak solutions of problems (1.1)-(1.2) and (1.1)-(1.3) we understand critical points of the associated energy functional,

$$
\mathcal{F}(u)=\frac{1}{2} \int_{\Omega}|\Delta u|^{2}+|u|^{2} d x-\int_{\partial \Omega} F(x, u) d \sigma
$$

where $\frac{\partial F}{\partial u}(x, u)=f(x, u)$.

This functional has to be set in $H=\left\{u \in H^{2}(\Omega): \frac{\partial u}{\partial \nu}=0\right.$ on $\left.\partial \Omega\right\}$ for the boundary condition (1.3) and in $H^{2}(\Omega)$ for (1.2). Throughout this paper we will consider the norm in $H^{2}(\Omega)$ given by $\|u\|_{H^{2}(\Omega)}^{2}=\|\Delta u\|_{L^{2}(\Omega)}^{2}+\|u\|_{L^{2}(\Omega)}^{2}$.

For (1.1)-(1.3) integration by parts shows that critical points of $\mathcal{F}$ are weak solutions of the problem. But, for (1.1)-(1.2) it is not immediate why the boundary condition (1.2) is verified, so we need to show further regularity on critical points to ensure this boundary condition. This is performed in $\S 2$.

In $\S 3$ we assume that $f(x, u)$ is superlinear and subcritical. The precise assumptions on the nonlinearity $f$ are the following:

$$
\begin{gathered}
|f(x, u)| \leq C\left(|u|^{p}+1\right), \\
F(x, u)=F(x,-u)
\end{gathered}
$$

and for $r>0$ small, if $|u| \leq r$,

$$
|F(x, u)| \leq C|u|^{\alpha}
$$

where $\alpha>2$ and $p$ satisfies

$$
1<p<\frac{N+2}{N-4}
$$

if $N>4$. For $|u|>R$ large enough,

$$
F(x, u) \leq \frac{1}{p+1} f(x, u) u
$$

This implies (cf. [8])

$$
F(x, u) \geq C|u|^{p+1}-C
$$

Our first result is:

Theorem 1.1. Let us assume that $f: \partial \Omega \times \mathbb{R} \rightarrow \mathbb{R}$ verifies (1.5)-(1.9). Then there exist infinitely many nontrivial solutions to (1.1)-(1.2) and infinitely many nontrivial solutions to (1.1)-(1.3).

In $\S 4$ we arrive at the main point of the paper, the critical case. More precisely, we assume that $f$ is a sublinear perturbation of the critical power in the following sense

$$
f(x, u)=|u|^{\frac{6}{N-4}} u+\lambda|u|^{q-1} u \quad \text { where } 0<q<1 .
$$


We remark that $\frac{6}{N-4}+1=\frac{N+2}{N-4}$ which is the critical exponent for our problem in the sense that $p=\frac{N+2}{N-4}=\frac{6}{N-4}+1$ is the critical exponent corresponding to the inclusion $H^{2}(\Omega) \hookrightarrow L^{p+1}(\partial \Omega)$. In this case we have the following result:

Theorem 1.2. Let $f$ satisfies (1.11) then there exists a constant $A$ such that if $0<$ $\lambda<A$, problem (1.1)-(1.2) and problem (1.1)-(1.3) have infinitely many nontrivial solutions.

The paper is organized as follows, in $\S 2$ we prove a regularity result for critical points of $\mathcal{F}$ that enables us to show that the boundary condition (1.2) is satisfied. In $\S 3$, we prove the existence of infinitely many critical points in the subcritical case (Theorem 1.1). Finally in $\S 4$ we deal with the critical case (Theorem 1.2).

\section{A Regularity Result}

In this section we prove a regularity result for critical points of $\mathcal{F}$ in $H^{2}(\Omega)$. First we observe that if $u$ is a critical point of $\mathcal{F}$ then it verifies

$$
0=\int_{\Omega} \Delta u \Delta \phi+u \phi d x-\int_{\partial \Omega} f(x, u) \phi d \sigma .
$$

As $u$ is in $H^{2}(\Omega)$, by our hypothesis on $f(1.5)-(1.9)$ and the Sobolev trace Theorem (cf. [1]) we have that $f(x, u)$ is in $L^{r}(\partial \Omega)$ with $r=\frac{2(N-1)}{p(N-4)} \geq 1$. Therefore $v=\Delta u$ is a weak solution of

$$
\left\{\begin{aligned}
-\Delta v & =u & & \text { in } \Omega \\
\frac{\partial v}{\partial \nu} & =-f(x, u) & & \text { on } \partial \Omega .
\end{aligned}\right.
$$

By standard regularity theory (cf. [9]) we have that $v=\Delta u \in W^{1, r}(\Omega)$, hence $\Delta u$ has a trace on $\partial \Omega$ and by integration by parts in (2.1) we conclude that $\Delta u=0$ in the sense of traces on $\partial \Omega$. Hence we have proved the following result:

Theorem 2.1. Every critical point of $\mathcal{F}$ in $H^{2}(\Omega)$ belongs to $W^{3, r}(\Omega), r=\frac{2(N-1)}{p(N-4)}$, and verifies $\left.\Delta u\right|_{\partial \Omega} \equiv 0$ in the sense of traces.

\section{Proof of Theorem 1.1}

In this section we consider problems (1.1)-(1.2) and (1.1)-(1.3) with $f$ subcritical, i.e. under hypotheses (1.5)-(1.9). The proof of Theorem 1.2 is an immediate consequence of a rather general Theorem due to [2]. We make only a sketch of the proof. Let us begin by checking the Palais-Smale condition.

Proposition 3.1. $\mathcal{F}$ verifies the Palais-Smale condition.

Proof. First, we consider $\mathcal{F}$ defined in $H^{2}(\Omega)$. Let $\left(u_{n}\right)_{n \geq 1} \subset H^{2}(\Omega)$ be a PalaisSmale sequence, that is

$$
\left|\mathcal{F}\left(u_{n}\right)\right| \leq c \quad \text { and } \quad \mathcal{F}^{\prime}\left(u_{n}\right) \rightarrow 0 .
$$

Let us first prove that $(3.1)$ implies that $\left(u_{n}\right)$ is bounded in $H^{2}$-norm. In fact, from (3.1) it follows that there exists a sequence $\varepsilon_{n} \rightarrow 0$ such that

$$
\left|\mathcal{F}^{\prime}\left(u_{n}\right) w\right| \leq \varepsilon_{n}\|w\|_{H^{2}(\Omega)}, \forall w \in H^{2}(\Omega)
$$


Now, using (3.1),

$$
\begin{aligned}
& c+\frac{1}{p+1} \varepsilon_{n}\left\|u_{n}\right\|_{H^{2}(\Omega)} \geq \mathcal{F}\left(u_{n}\right)-\frac{1}{p+1} \mathcal{F}^{\prime}\left(u_{n}\right) u_{n} \\
& =\left(\frac{1}{2}-\frac{1}{p+1}\right) \int_{\Omega}\left|\Delta u_{n}\right|^{2}+\left|u_{n}\right|^{2} d x-\int_{\partial \Omega} F\left(u_{n}\right)-\frac{1}{p+1} f\left(u_{n}\right) u_{n} d \sigma .
\end{aligned}
$$

From (1.9) and (3.3) we obtain $c\left(1+\left\|u_{n}\right\|_{H^{2}(\Omega)}\right) \geq\left\|u_{n}\right\|_{H^{2}(\Omega)}^{2}$ and then, $u_{n}$ is bounded in $H^{2}(\Omega)$. Now the proof follows using standard arguments, using the subcriticality assumption on $f,(1.8)$. The case where $\mathcal{F}$ is defined in $H=\{u \in$ $\left.H^{2}(\Omega): \partial u / \partial \nu=0\right\}$ can be handled in an analogous way.

Now we recall a topological tool, the genus, that was introduced in [11] but we will use an equivalent definition due to [5]. Given a Banach Space $X$, we consider the class

$$
\Sigma=\{A \subset X: A \text { is closed, } A=-A\} .
$$

Over this class we define the genus, $\gamma: \Sigma \rightarrow \mathbb{N} \cup\{\infty\}$, as

$$
\gamma(A)=\min \left\{k \in \mathbb{N}: \text { there exists } \varphi \in C\left(A, \mathbb{R}^{k}-\{0\}\right), \varphi(x)=-\varphi(-x)\right\} .
$$

We need the following properties of the genus.

Proposition 3.2. ([16], Proposition 7.5) Let $A, B \in \Sigma$. Then

(1) If there exists an odd map $f \in C(A, B)$, then $\gamma(A) \leq \gamma(B)$.

(2) If $A \subset B$, then $\gamma(A) \leq \gamma(B)$.

(3) $\gamma(A \cup B) \leq \gamma(A)+\gamma(B)$.

(4) If $A$ is compact, then $\gamma(A)<\infty$ and there exists $\delta>0$ such that $\gamma\left(N_{\delta}(A)\right)=$ $\gamma(A)$, where $N_{\delta}(A)=A+\overline{B_{\delta}(0)}$.

For the proof of Theorem 1.1, we will use the following Theorem due to [2],

Theorem 3.1. ([2], Theorem 2.23) Let $\mathcal{F}: X \rightarrow \mathbb{R}$ verifying

(1) $\mathcal{F} \in C^{1}(X)$ and even.

(2) $\mathcal{F}$ verifies the Palais-Smale condition.

(3) There exists a constant $r>0$ such that $\mathcal{F}(u)>0$ in $0<\|u\|_{X}<r$, and $\mathcal{F}(u) \geq c>0$ if $\|u\|_{X}=r$.

(4) There exists a closed subspace $E_{m} \subset X$ of dimension m, and a compact set $A_{m} \subset E_{m}$ such that $\mathcal{F}<0$ on $A_{m}$ and 0 lies in a bounded component of $E_{m}-A_{m}$ in $E_{m}$.

Let

$\Gamma=\{h \in C(X, X): h(0)=0, h$ is an odd homeomorphism and $\mathcal{F}(h(B)) \geq 0\}$,

and

$\mathcal{K}_{m}=\{K \subset X: K=-K, K$ is compact, and $\gamma(K \cap h(\partial B)) \geq m$ for all $h \in \Gamma\}$, where $B$ is the unit ball. Then,

$$
c_{m}=\inf _{K \in \mathcal{K}_{m}} \max _{u \in K} \mathcal{F}(u)
$$

is a critical value of $\mathcal{F}$, with $0<c \leq c_{m} \leq c_{m+1}<\infty$. Moreover, if $c_{m}=c_{m+1}=$ $\cdots=c_{m+r}$ then $\gamma\left(K_{c_{m}}\right) \geq r+1$ where $K_{c_{m}}=\left\{u \in X: \mathcal{F}^{\prime}(u)=0, \mathcal{F}(u)=c_{m}\right\}$. 
With this result, we are able to prove Theorem 1.1.

Proof of Theorem 1.1: We deal with the functional $\mathcal{F}$ in $H^{2}(\Omega)$. The other boundary condition can be handled in a similar fashion.

We have to check the hypotheses of Theorem 3.1. The fact that $\mathcal{F}$ is $C^{1}$ and even is a consequence of our hypotheses on $f(1.5)-(1.6)$ (see [16] for details). The Palais-Smale condition was already checked in Lemma 3.1.

To verify 3 let us consider, for a fixed $u \in H^{2}(\Omega),\|u\|_{H^{2}(\Omega)}=1$ and $t$ small, using (1.7) and the Sobolev trace Theorem,

$$
g(t):=\mathcal{F}(t u)=\frac{t^{2}}{2}-\int_{\partial \Omega} F(t u) d \sigma \geq \frac{t^{2}}{2}-C t^{\alpha} .
$$

As $\alpha>2$, we have that $g(t) \geq \delta>0$ if $t<t_{0}$.

Finally, we have to check 4 . We consider subspaces $E_{m} \subset E_{m+1} \subset H^{2}(\Omega)$ such that $u \neq \equiv 0$ on $\partial \Omega$ for every $u \in E_{m}, u \neq 0$ in the sense of traces. We observe that, as $\operatorname{dim} E_{m}<\infty$, then

$$
\min _{\left\{u \in E_{m},\|u\|_{H^{2}(\Omega)}=1\right\}} \int_{\partial \Omega} u^{p+1} d \sigma>0 .
$$

As $A_{m}$ we take $\left\{u \in E_{m}:\|u\|_{H^{2}(\Omega)}=R\right\}$. Now, if $u \in A_{m}$ then, by (1.10),

$$
\mathcal{F}(u)=\frac{R^{2}}{2}-\int_{\partial \Omega} F(u) d \sigma \leq \frac{R^{2}}{2}-C R^{p+1} \min _{\left\{u \in E_{m},\|u\|_{H^{2}(\Omega)}=1\right\}} \int_{\partial \Omega} u^{p+1} d \sigma+C
$$

therefore, if we choose $R$ large enough, we have 4 .

\section{Proof of Theorem 1.2}

In this section we study the critical case with a sublinear perturbation, i.e. $f$ verifies (1.11), that is $f(x, u)=|u|^{\frac{6}{N-4}} u+\lambda|u|^{q-1} u$ where $0<q<1$. The technical result used here, the concentrated compactness method, is mainly due to [12], [13]. See [3] for the concentration compactness principle applied to a fourth order semilinear problem. Throughout this section, we will call $E$ the space $H^{2}(\Omega)$ or $H=\left\{u \in H^{2}(\Omega): \frac{\partial u}{\partial \nu}=0\right\}$. As before we are considering any of the two boundary conditions (1.3) or (1.2).

To prove our existence result, since we have lost the compactness in the inclusion $H^{2}(\Omega) \hookrightarrow L^{p+1}(\partial \Omega)$, we can no longer expect the Palais-Smale condition to hold. Anyway we can prove a local Palais-Smale condition that will hold for $\mathcal{F}(u)$ below a certain value of energy.

Let $u_{j}$ be a bounded sequence in $E$ then there exists a subsequence, that we still denote $u_{j}$, such that $u_{j} \rightarrow u$ weakly in $E, \Delta u_{j} \rightarrow d \mu,\left.\left|u_{j}\right|_{\partial \Omega}\right|^{\frac{2 N-2}{N-4}} \rightarrow d \eta$, weakly-* in the sense of measures.

If we consider $\phi \in C^{\infty}(\bar{\Omega})$, from the Sobolev trace inequality we obtain

$$
\begin{gathered}
\left(\int_{\partial \Omega}|\phi|^{\frac{2 N-2}{N-4}} d \eta\right)^{\frac{N-4}{2 N-2}} S^{1 / 2} \leq\left(\int_{\Omega}|\phi|^{2} d \mu\right)^{1 / 2}+2\left(\int_{\Omega}\langle\nabla \phi \nabla u\rangle^{2} d x\right)^{1 / 2}+ \\
\left(\int_{\Omega}|\Delta \phi|^{2}|u|^{2} d x\right)^{1 / 2}+\left(\int_{\Omega}|\phi|^{2}|u|^{2} d x\right)^{1 / 2},
\end{gathered}
$$

where $S$ is the best constant in the Sobolev trace embedding Theorem.

¿From (4.1), we have that, if $u=0$ we get a reverse Holder type inequality (it involves one integral over $\partial \Omega$ and one over $\Omega$ ) between the two measures $\mu$ and $\eta$. 
Now we state the following Lemma due to [12], [13].

Lemma 4.1. ([12], [13]) Let $u_{j}$ be a weakly convergent sequence in E with weak limit $u$ such that $\Delta u_{j} \rightarrow d \mu$ and $\left.\left|u_{j}\right|_{\partial \Omega}\right|^{\frac{2 N-2}{N-4}} \rightarrow d \eta$, weakly- ${ }^{*}$ in the sense of measures. Then there exists $x_{1}, \ldots, x_{l} \in \partial \Omega$ such that

(1) $\eta=|u|^{\frac{2 N-2}{N-4}}+\sum_{j=1}^{l} \eta_{j} \delta_{x_{j}}, \eta_{j}>0$,

(2) $\mu \geq|\Delta u|^{2}+\sum_{j=1}^{l} \mu_{j} \delta_{x_{j}}, \mu_{j}>0$,

(3) $\left(\eta_{j}\right)^{\frac{N-4}{N-1}} \leq \frac{\mu_{j}}{S}$

Next, we use Lemma 4.1 to prove a local Palais-Smale condition.

Lemma 4.2. Let $u_{j} \subset E$ be a Palais-Smale sequence for $\mathcal{F}$, with energy level $c$. If $c<\frac{3}{2 N-2} S^{\frac{N-1}{3}}-K \lambda^{\frac{2 N-2}{N+4-(N-4) q}}$, where $K$ depends only on $q, N$, and $|\partial \Omega|$, then there exists a subsequence $u_{j_{k}}$ that converges strongly in $E$.

Proof. From the fact that $u_{j}$ is a Palais-Smale sequence it follows that $u_{j}$ is bounded in $E$ (see Lemma 3.1). By Lemma 4.1 there exists a subsequence, that we still denote $u_{j}$, such that

$$
\begin{aligned}
& u_{j} \rightarrow u \text { weakly in } E, \\
& u_{j} \rightarrow u \text { in } L^{r}(\partial \Omega), 1<r<\frac{2 N-2}{N-4}, \text { and a.e. in } \partial \Omega, \\
& \left|\Delta u_{j}\right|^{2} \rightarrow d \mu \geq|\Delta u|^{2}+\sum_{k=1}^{l} \mu_{k} \delta_{x_{k}}, \\
& \left.\left|u_{j}\right|_{\partial \Omega}\right|^{\frac{2 N-2}{N-4}} \rightarrow d \eta=\left.|u|_{\partial \Omega}\right|^{\frac{2 N-2}{N-4}}+\sum_{k=1}^{l} \eta_{k} \delta_{x_{k}} .
\end{aligned}
$$

Let $\phi \in C^{\infty}\left(\mathbb{R}^{N}\right)$ such that $\phi \equiv 1$ in $B\left(x_{k}, \varepsilon\right), \phi \equiv 0$ in $B\left(x_{k}, 2 \varepsilon\right)^{c},|\nabla \phi| \leq 2 / \varepsilon$ and $|\Delta \phi| \leq 2 / \varepsilon^{2}$, where $x_{k}$ is a point at $\partial \Omega$ that belongs to the support of $d \eta$. Consider $\left(u_{j} \phi\right)$. Obviously this sequence is bounded in $E$. As $\mathcal{F}^{\prime}\left(u_{j}\right) \rightarrow 0$, we obtain that

$$
\int_{\partial \Omega} \phi d \eta+\lambda \int_{\partial \Omega}|u|^{q+1} \phi d \sigma=\int_{\Omega}|u|^{2} \phi d x+\lim _{j \rightarrow \infty} \int_{\Omega} \Delta u_{j} \Delta\left(u_{j} \phi\right) d x .
$$

By (4.2) we obtain,

$$
\lim _{j \rightarrow \infty} \int_{\Omega} \Delta u_{j} \Delta\left(u_{j} \phi\right) d x=\int_{\Omega} \phi d \mu+\lim _{j \rightarrow \infty} \int_{\Omega} \Delta u_{j}\left(2 \nabla u_{j} \nabla \phi+u_{j} \Delta \phi\right) d x .
$$

Now, by Hölder inequality and weak convergence,

$$
\begin{aligned}
0 & \leq \lim _{j \rightarrow \infty}\left|\int_{\Omega} \Delta u_{j} \nabla u_{j} \nabla \phi d x\right| \leq \lim _{j \rightarrow \infty}\left(\int_{\Omega}\left|\Delta u_{j}\right|^{2} d x\right)^{1 / 2}\left(\int_{\Omega}|\nabla \phi|^{2}\left|\nabla u_{j}\right|^{2} d x\right)^{1 / 2} \\
& \leq C\left(\int_{B\left(x_{k}, \varepsilon\right) \cap \Omega}|\nabla \phi|^{2}|\nabla u|^{2} d x\right)^{1 / 2} \\
& \leq C\left(\int_{B\left(x_{k}, \varepsilon\right) \cap \Omega}|\nabla \phi|^{N} d x\right)^{1 / N}\left(\int_{B\left(x_{k}, \varepsilon\right) \cap \Omega}|\nabla u|^{2 N /(N-2)} d x\right)^{(N-2) / 2 N} \\
& \leq C\left(\int_{B\left(x_{k}, \varepsilon\right) \cap \Omega}|\nabla u|^{2 N /(N-2)} d x\right)^{(N-2) / 2 N} \rightarrow 0, \quad \text { as } \varepsilon \rightarrow 0 .
\end{aligned}
$$


Analogously, we obtain

$$
0 \leq \lim _{j \rightarrow \infty}\left|\int_{\Omega} \Delta u_{j} u_{j} \Delta \phi d x\right| \leq C\left(\int_{B\left(x_{k}, \varepsilon\right) \cap \Omega}|u|^{2 N /(N-4)} d x\right)^{(N-4) / 2 N} \rightarrow 0,
$$

as $\varepsilon \rightarrow 0$. Then

$$
\lim _{\varepsilon \rightarrow 0}\left[\int_{\partial \Omega} \phi d \eta+\lambda \int_{\partial \Omega}|u|^{q} \phi d \sigma-\int_{\Omega} \phi d \mu-\int_{\Omega}|u|^{2} \phi d x\right]=\eta_{k}-\mu_{k}=0 .
$$

By Lemma 4.1 we have that $\left(\eta_{k}\right)^{\frac{N-4}{N-1}} S \leq \mu_{k}$, hence by $(4.3)$ we obtain $\left(\eta_{k}\right)^{\frac{N-4}{N-1}} S \leq$ $\eta_{k}$. Then, either $\eta_{k}=0$ or

$$
\eta_{k} \geq S^{\frac{N-1}{3}}
$$

If (4.4) does indeed occurs for some $k_{0}$ then, from the fact that $u_{j}$ is a Palais-Smale sequence, we obtain

$$
\begin{gathered}
c=\lim _{j \rightarrow \infty} \mathcal{F}\left(u_{j}\right)=\lim _{j \rightarrow \infty}\left(\mathcal{F}\left(u_{j}\right)-\mathcal{F}^{\prime}\left(u_{j}\right) u_{j}\right) \geq \\
\frac{3}{2 N-2} \int_{\partial \Omega}|u|^{\frac{2 N-2}{N-4}} d \sigma+\frac{3}{2 N-2} S^{\frac{N-1}{3}}-\lambda\left(\frac{1}{q+1}-\frac{1}{2}\right) \int_{\partial \Omega}|u|^{q+1} d \sigma .
\end{gathered}
$$

As $0<q<1$, we can apply Hölder inequality in (4.5) to obtain

$$
\begin{gathered}
c \geq \frac{3}{2 N-2} \int_{\partial \Omega}|u|^{\frac{2 N-2}{N-4}} d \sigma+\frac{3}{2 N-2} S^{\frac{N-1}{3}}- \\
\lambda\left(\frac{1}{q+1}-\frac{1}{2}\right)|\partial \Omega|^{\frac{N+4-(N-4) q}{2 N-2}}\left(\int_{\partial \Omega}|u|^{\frac{2 N-2}{N-4}} d \sigma\right)^{\frac{(q+1)(N-4)}{2 N-2}} .
\end{gathered}
$$

Let $\varphi(t)=c_{1}+c_{2} t^{\frac{2 N-2}{N-4}}-\lambda c_{3} t^{q+1}$. The minimum of $\varphi$ is attained at $t_{0}=$ $\left(\frac{\lambda c_{3}(q+1)(N-4)}{(2 N-2) c_{2}}\right)^{\frac{N-4}{N+4-(N-4) q}} \cdot$ Therefore,

$$
\varphi(t) \geq \varphi\left(t_{0}\right)=c_{1}+c_{2}\left(t_{0}\right)^{\frac{N-4}{2 N-2}}-\lambda c_{3}\left(t_{0}\right)^{q+1}=\frac{3}{2 N-2} S^{\frac{N-1}{3}}-K \lambda^{\frac{2 N-2}{N+4-(N-4) q}},
$$

which contradicts the hypothesis that $c<\frac{3}{2 N-2} S^{\frac{N-1}{3}}-K \lambda^{\frac{2 N-2}{N+4-(N-4) q}}$. This implies that $\eta_{k}=0$ for every $k$, therefore, by (4.2), we have that $\left\|u_{j}\right\|_{H^{2}(\Omega)} \rightarrow\|u\|_{H^{2}(\Omega)}$. This fact and the weak convergence end the proof.

We observe, using the trace Theorem, that

$$
\mathcal{F}(u) \geq \frac{1}{2}\|u\|_{H^{2}(\Omega)}^{2}-c_{1}\|u\|_{H^{2}(\Omega)}^{\frac{2 N-2}{N-4}}-\lambda c_{2}\|u\|_{H^{2}(\Omega)}^{q+1}=j\left(\|u\|_{H^{2}(\Omega)}\right)
$$

where $j(x)=\frac{1}{2} x^{2}-c_{1} x^{\frac{2 N-2}{N-4}}-\lambda c_{2} x^{q+1}$. As $j$ attains a local but not a global minimum ( $j$ is not bounded below), we have to perform some sort of truncation. To this end let $x_{0}, x_{1}$ be such that $m<x_{0}<M<x_{1}$ where $m$ is the local minimum of $j$ and $M$ is the local maximum and $j\left(x_{1}\right)>j(m)$. For these values $x_{0}$ and $x_{1}$ we can choose a smooth function $\tau(x)$ such that $\tau(x)=1$ if $x \leq x_{0}, \tau(x)=0$ if $x \geq x_{1}$ and $0 \leq \tau(x) \leq 1$. Finally, let $\varphi(u)=\tau\left(\|u\|_{H^{2}(\Omega)}\right)$ and define the truncated functional as follows

$$
\tilde{\mathcal{F}}(u)=\frac{1}{2} \int_{\Omega}|\Delta u|^{2}+|u|^{2} d x-\frac{N-4}{2 N-2} \int_{\partial \Omega}|u|^{\frac{2 N-2}{N-4}} \varphi(u) d \sigma-\lambda \frac{1}{q+1} \int_{\partial \Omega}|u|^{q+1} d \sigma .
$$


As above, $\tilde{\mathcal{F}}(u) \geq \tilde{j}\left(\|u\|_{H^{2}(\Omega)}\right)$ where $\tilde{j}(x)=\frac{1}{2} x^{2}-c_{1} x^{\frac{2 N-2}{N-4}} \tau(x)-\lambda c_{2} x^{q+1}$. We observe that if $x \leq x_{0}$ then $\tilde{j}(x)=j(x)$ and if $x \geq x_{1}$ then $\tilde{j}(x)=\frac{1}{2} x^{2}-\lambda c_{2} x^{q+1}$.

Now we state a Lemma that contains the main properties of $\tilde{\mathcal{F}}$.

Lemma 4.3. $\tilde{\mathcal{F}}$ is $C^{1}$, if $\tilde{\mathcal{F}}(u) \leq 0$ then $\|u\|_{H^{2}(\Omega)}<x_{0}$ and $\mathcal{F}(v)=\tilde{\mathcal{F}}(v)$ for every $v$ close enough to $u$. Moreover there exists $A=A(q, N, \Omega)>0$ such that if $0<\lambda<A$ then $\tilde{\mathcal{F}}$ satisfies a local Palais-Smale condition for $c \leq 0$.

Proof. We only have to check the local Palais-Smale condition. Observe that every Palais-Smale sequence for $\tilde{\mathcal{F}}$ with energy level $c \leq 0$ must be bounded, therefore by Lemma 4.2 if $\lambda$ verifies $0<\frac{3}{2 N-2} S^{\frac{N-1}{3}}-K \lambda^{\frac{2 N-2}{N+4-(N-4) q}}$ then there exists a convergent subsequence.

Next Lemma gives the final ingredient needed in the proof of Theorem 1.2.

Lemma 4.4. For every $n \in \mathbb{N}$ there exists $\varepsilon>0$ such that $\gamma\left(\tilde{\mathcal{F}}^{-\varepsilon}\right) \geq n$, where $\tilde{\mathcal{F}}^{-\varepsilon}=\{u, \tilde{\mathcal{F}}(u) \leq-\varepsilon\}$.

Proof. Fix $n \in \mathbb{N}$ and choose $E_{n}$ an $n$-dimensional subspace such that $\left.u\right|_{\partial \Omega} \not \equiv 0$ if $u \in E_{n}, u \not \equiv 0$. Therefore,

$$
\begin{aligned}
& \alpha_{n}=\inf \left\{\int_{\partial \Omega}|u|^{\frac{N+2}{N-4}} d \sigma, u \in E_{n},\|u\|_{H^{2}(\Omega)}=1\right\}>0, \\
& \beta_{n}=\inf \left\{\int_{\partial \Omega}|u|^{q+1} d \sigma, u \in E_{n},\|u\|_{H^{2}(\Omega)}=1\right\}>0 .
\end{aligned}
$$

Now take $u \in E_{n}$ with $\|u\|_{H^{2}(\Omega)}=1$, then for $0<\rho<x_{0}$ it holds

$$
\tilde{\mathcal{F}}(\rho u)=\mathcal{F}(\rho u) \leq \frac{1}{2} \rho^{2}-\alpha_{n} \frac{N-4}{N+2} \rho^{\frac{N+2}{N-4}}-\beta_{n} \frac{\lambda}{q+1} \rho^{q+1} .
$$

As $q<1$ we can choose $\varepsilon=\varepsilon(\rho)>0$ such that $\tilde{\mathcal{F}}(\rho u) \leq-\varepsilon$ if $u \in E_{n},\|u\|_{H^{2}(\Omega)}=1$. Therefore, by the monotonicity of the genus, $\gamma\left(\tilde{\mathcal{F}}^{-\varepsilon}\right) \geq \gamma\left(\partial B(0, \rho) \cap E_{n}\right)=n$.

Finally, we are ready to prove the main result of the paper.

\section{Proof of Theorem 1.2 Let}

$$
\Sigma_{k}=\left\{C \subset H^{2}(\Omega)-\{0\}, C \text { is closed }, C=-C, \gamma(C) \geq k\right\}
$$

and

$$
c_{k}=\inf _{C \in \Sigma_{k}} \sup _{u \in C} \tilde{\mathcal{F}}(u), \quad K_{c}=\left\{u \in H^{2}(\Omega), \tilde{\mathcal{F}}^{\prime}(u)=0, \tilde{\mathcal{F}}(u)=c\right\} .
$$

We want to show that if $c=c_{k}=c_{k+1}=\cdots=c_{k+r}$ then $\gamma\left(K_{c}\right) \geq r+1$. With this purpose, we first prove that $-\infty<c_{k}<0$. In fact, by Lemma 4.4 there exists $\varepsilon=\varepsilon(k)>0$ such that $\gamma\left(\tilde{\mathcal{F}}^{-\varepsilon}\right) \geq k$. Now as $\tilde{\mathcal{F}}$ is continuous and even, $\tilde{\mathcal{F}}^{-\varepsilon} \in \Sigma_{k}$ then $c_{k} \leq-\varepsilon<0$. As $\tilde{\mathcal{F}}$ is bounded from below it follows that $c_{k}>-\infty$.

Let us assume by contradiction that $c=c_{k}=\cdots=c_{k+r}$ and $\gamma\left(K_{c}\right)<r+1$. Observe that as $c<0, \tilde{\mathcal{F}}$ verifies the Palais-Smale condition in $K_{c}$ and therefore $K_{c}$ is compact. Also it is symmetric. Now, by the deformation Lemma (cf. [16]), there exists an odd homeomorphism $\eta: H^{2}(\Omega) \rightarrow H^{2}(\Omega)$ such that $\eta\left(\tilde{\mathcal{F}}^{c+\delta}-K_{c}\right) \subset \tilde{\mathcal{F}}^{c-\delta}$ and one can easily check that we must choose $0<\delta<-c$ because $\tilde{\mathcal{F}}$ verifies the Palais-Smale condition on $\tilde{\mathcal{F}}^{0}$. We have $c=c_{k+r}=\inf _{C \in \Sigma_{k+r}} \sup _{u \in C} \tilde{\mathcal{F}}(u)$. Hence there exists $C \in \Sigma_{k+r}$ such that $\sup _{u \in C} \tilde{\mathcal{F}}(u) \leq c+\delta$ and therefore

$$
\eta\left(C-K_{c}\right) \subset \eta\left(\tilde{\mathcal{F}}^{c+\delta}-K_{c}\right) \subset \tilde{\mathcal{F}}^{c-\delta} .
$$


But $\gamma\left(\overline{C-K_{c}}\right) \geq \gamma(C)-\gamma\left(K_{c}\right) \geq k$ and $\gamma\left(\overline{\eta\left(C-K_{c}\right)}\right) \geq \gamma\left(\overline{C-K_{c}}\right) \geq k$. Therefore $\overline{\eta\left(C-K_{c}\right)} \in \Sigma_{k}$, since $\overline{\eta\left(C-K_{c}\right)} \in \Sigma_{k}$ implies $\sup _{u \in \overline{\eta\left(C-K_{c}\right)}} \tilde{\mathcal{F}}(u) \geq c_{k}=c$, this contradicts $(4.7)$. We get infinitely many critical points of $\tilde{\mathcal{F}}$ with negative energy and hence, by Lemma 4.3 , these points are critical points of $\mathcal{F}$. This shows the existence of infinitely many weak solutions of (1.1)-(1.2).

Acknowledgements: We want to thank L. D'Alfonso for several suggestions improving our manuscript.

\section{REFERENCES}

[1] R.A. Adams, Sobolev spaces, Academic Press, New York, 1975.

[2] A. Ambrosetti and P.H. Rabinowitz, Dual variational methods in critical point theory and applications, J. Funct. Anal., Vol. 14(4) (1973), 349-381.

[3] F. Bernis, J. Garcia-Azorero and I. Peral, Existence and multiplicity of nontrivial solutions in semilinear critical problems of fourth order, Adv. Differ. Equ. 1, No.2 (1996), 219-240.

[4] M. Chipot, I. Shafrir and M. Fila, On the solutions to some elliptic equations with nonlinear Neumann boundary conditions, Adv. Differ. Equ. 1, No.1 (1996), 91-110.

[5] C.V. Coffman, A minimum-maximum principle for a class of nonlinear integral equations, J. Analyse Math., 22 (1969), 391-419.

[6] D.G. de Figueiredo, Semilinear elliptic systems: a survey of superlinear problems, Resenhas IME-USP, 2 (1996), 373-391.

[7] D.E. Edmunds, D. Fortunato and E. Janelli, Critical exponents, critical dimension and the biharmonic operator, Arch. Rational Mech. Anal. 112 (1990), 269-289.

[8] P. Felmer, Periodic solutions of 'superquadratic' Hamiltonian systems, J. Diff. Eq., 102 (1993), 188-207.

[9] D. Gilbarg and N. S. Trudinger, Elliptic Partial Differential Equations of Second Order, Springer-Verlag, NY (1983).

[10] B. Hu, Non existence of a positive solution of the Laplace equation with a nonlinear boundary condition, Diff. and Int. Equ., 7 (1994), 301-313.

[11] M.A. Krasnoselski, Topological methods in the theory of nonlinear integral equations, Macmillan, New York, 1964.

[12] P.L. Lions, The concentration-compactness principle in the calculus of variations. The limit case, part 1, Rev. Mat. Iberoamericana 1 No.1 (1985), 145-201.

[13] P.L. Lions, The concentration-compactness principle in the calculus of variations. The limit case, part 2, Rev. Mat. Iberoamericana 1 No.2 (1985), 45-121.

[14] E.S. Noussair, C.A. Swanson and Y. Jianfu, Critical semilinear biharmonic equations in $\mathbb{R}^{N}$, Proc. Royal Soc. Edinburgh 121 A (1992), 139-148.

[15] P. Pucci and J. Serrin, Critical exponents and critical dimensions for polyharmonic operators, J. Math. Pures Appl. 69 (1990), 55-83.

[16] P. Rabinowitz, Minimax methods in critical point theory with applications to differential equations, CBMS Reg. Conf. Ser. Math., no. 65, Amer. Math. Soc., Prov., R.I. (1986).

Departamento de Matemática, FCEyn, ubA (1428) Buenos Aires, Argentina.

E-mail address: jfbonder@dm.uba.ar, jrossi@dm.uba.ar 